Results In line with previous reports for CAR-T cells, dasatinib (a src inhibitor) was found to fully switch off TCB-induced T cell functionality as well as the other src inhibitors bosutinib and ponatinib. In contrast, temsirolimus, sirolimus and everolimus (mTOR inhibitors) and ruxolitinib, baricitinib, tofacitinib, and fedratinib (JAK1/2 inhibitors) were found to more potently prevent TCB-induced cytokine release without blocking TCB-mediated target cell killing.

Conclusions These results provide evidence that the mechanisms of TCB-dependent cytokine release and tumor cell killing can be uncoupled. The FDA-approved mTOR and JAK1/2 inhibitors could potentially be used to mitigate CRS whereas the Src inhibitor dasatinib could rather stand as a potential antidote for on-target off-tumor activity or high-grade CRS.

Disclosure Information G. Leclercq: A. Employment (full or part-time); Modest; Roche. E. Ownership Interest (stock, stock options, patent or other intellectual property); Modest; Roche. H. Haegel: A. Employment (full or part-time); Modest; Roche. E. Ownership Interest (stock, stock options, patent or other intellectual property); Modest; Roche. A. Schneider: A. Employment (full or part-time); Modest; Roche. A. Giusti: A. Employment (full or part-time); Modest; Roche. V. Pulko: A. Employment (full or part-time); Modest; Roche. A. Toso: A. Employment (full or part-time); Modest; Roche. T. Zimmermann: A. Employment (full or part-time); Modest; Roche. L. Green: A. Employment (full or part-time); Modest; Roche. N. Steinhoff: A. Employment (full or part-time); Modest; Roche. J. Sam: A. Employment (full or part-time); Modest; Roche. M. Bacac: A. Employment (full or part-time); Modest; Roche. E. Ownership Interest (stock, stock options, patent or other intellectual property); Modest; Roche. P. Umaña: A. Employment (full or part-time); Modest; Roche. E. Ownership Interest (stock, stock options, patent or other intellectual property); Modest; Roche. C. Klein: A. Employment (full or part-time); Modest; Roche. E. Ownership Interest (stock, stock options, patent or other intellectual property); Modest; Roche.

\section{P09.02 EPIGENETIC MODULATION OF NEUROBLASTOMA ENHANCES T- AND NK CELL IMMUNOGENICITY VIA INDUCTION OF SURFACE EXPRESSION OF MHC CLASS I AND MICA/MICB}

\footnotetext{
${ }^{1,2} \mathrm{AM}$ Cornel* ${ }^{*}{ }^{1,2} \mathrm{DAMH}$ van den Beemt, ${ }^{1,2} \mathrm{E}$ Dunnebach, ${ }^{1} \mathrm{MP}$ van Dierselhuis, 1,2S Nierkens. 'Princess Maxima Center, Utrecht, Netherlands; ${ }^{2}$ UMC Utrecht, Utrecht, Netherlands
}

10.1136/jitc-2021-ITOC8.52

Background Neuroblastoma (NBL) is the most common pediatric solid tumor and responsible for about $15 \%$ of all pediatric cancer deaths. The majority of high-risk (HR) patients suffers from relapse after intense therapy regimens, resulting in a 5 -year survival rate of only $40 \%$. Even though the potential of immune interference in HR-NBL is shown by the additive effect of anti-GD2 monoclonal antibody therapy to the treatment protocol, long-term follow-up studies reveal that the beneficial effect of immunotherapy diminishes over time. We hypothesize that this is a result of inadequate (adaptive) immune engagement caused by the extensive immunomodulatory capacity of HR-NBL and its microenvironment. One of the most remarkable immunomodulatory strategies of NBL tumors is the absence of MHC-I surface expression, thereby preventing cytotoxic $\mathrm{T}$ cell recognition and killing. MHC-I lacking cells are known to be subjected to NK cell mediated cytotoxicity, however, we have shown that NBL is able to evade this by temporary upregulating surface expression of MHC-I, thereby becoming temporarily more prone to $\mathrm{T}$ cell mediated cytotoxicity. The aim of this project is to identify pharmacological strategies to enhance adaptive immune activation and therewith immunogenicity of HR-NBL.

Materials and Methods FDA-approved drug libraries were screened to identify compounds enhancing MHC-I surface expression in NBL cell lines using high-throughput flow cytometry analyses optimized for adherent NBL cells. The effect of positive hits was subsequently confirmed in a panel of NBL patient-derived tumeroids. Alterations in the transcriptome and translatome upon incubation with compounds of interest were further studied to identify potential additional immunomodulatory effects in NBL. Ultimately, compound treated NBL cell lines and tumeroids were co-cultured with PRAME reactive tumor-specific $\mathrm{T}$ cells and healthy-donor NK cells to determine the in vitro effect on $\mathrm{T}$ - and $\mathrm{NK}$ cell cytotoxicity.

Results Drug library screening revealed MHC-I upregulation upon treatment of NBL cell lines and patient-derived tumeroids with multiple histon deacetylase inhibitors (HDACi). Further investigation of immunomodulatory effects of HDACi in NBL revealed enhanced expression of several additional players of the antigen presenting machinery, immunoproteasome expression, and MICA/MICB upregulation in NBL cells. We show that in untreated NBL cells, plasticity of MHC-I expression causes evasion of both NK- and T cell mediated cytotoxicity. Intriguingly, co-culture of NBL cells with tumor-specific $\mathrm{T}$ cells and healthy-donor NK cells upon treatment with the HDACi Entinostat resulted in enhanced in vitro T- and NK cell activation and cytotoxicity.

Conclusions We show pharmacological upregulation of MHCI, other antigen presenting machinery players, and the NKG2D ligands MICA/MICB upon HDACi in HR-NBL. Pretreatment of NBL with HDACi resulted in enhanced in vitro T- and NK cell mediated cytotoxicity, substantiating HDACi as a potential strategy to improve adaptive immune engagement and therewith immunogenicity to aid NBL treatment.

This work was supported by the Villa Joep Foundation [IWOV-Actief.51391.180034].

Disclosure Information A.M. Cornel: None. D.A.M.H. van den Beemt: None. E. Dunnebach: None. M.P. van Dierselhuis: None. S. Nierkens: None.

\section{P09.03 HYALURONIC ACID AS A NEW IMMUNOLOGIC ADJUVANT IN CANCER: DESIGN OF EFFECTIVE PREVENTIVE AND THERAPEUTIC VACCINATION STRATEGIES FOR HER2/NEU-POSITIVE BREAST TUMORS}

${ }^{1} \mathrm{D}$ Carpanese*, 'I Montagner, ${ }^{2} \mathrm{~A}$ Dalla Pietà, ${ }^{1} \mathrm{~V}$ Rossi, ${ }^{2} \mathrm{~A}$ Penna, ${ }^{2} \mathrm{G}$ Zuccolotto, ${ }^{2} \mathrm{G}$ Pasut, ${ }^{2} \mathrm{~A}$ Grigoletto, ${ }^{1} \mathrm{~A}$ Rosato. ${ }^{1}$ Veneto Institute of Oncology IOV-IRCCS, Padova, Italy; ${ }^{2}$ University of Padova, Padova, Italy

\subsection{6/jitc-2021-ITOC8.53}

Background The use of proteins as immunogens is attractive for the development of vaccines, but requires efficient adjuvants to overcome their weak immunogenicity. Recently, we investigated the potential of the TLR2/4 agonist hyaluronan (HA) as an immunological adjuvant for protein-based vaccines. ${ }^{1} 2$ Conjugation of $\mathrm{HA}$ to antigens strongly increased 
their immunogenicity and promoted their rapid translocation to draining lymph nodes, resulting in robust and long-lasting humoral responses. ${ }^{1}$ On these bases, we investigated the potentiality of HA-based technology in the design of cancer vaccines. To this aim, HA was conjugated to the extracellular domain of rat HER2/neu (rHER2/neu) and validated in the preventive and therapeutic vaccination settings.

Materials and Methods Female BALB/c or BALB-neuT mice were immunized with rHER2/neu-HA. In vivo depletion of $\mathrm{CD}^{+}, \mathrm{CD}^{+} \mathrm{T}$ and $\mathrm{B}$ cells was performed, and sera and spleens were collected to characterized antigen-specific humoral and cellular responses. Vaccinated BALB/c mice were challenged and re-challenged with rHER2/neu-overexpressing TUBO cells to assess the protective or therapeutic activity of rHER2/neu-HA vaccination strategy, as well as immunological memory.

Results HA performed efficiently as robust and long-lasting humoral (IgG1, IgG2a, and IgG2b) and cellular responses were detected using very low antigen doses and number of boosters. Outstandingly, at 1-year post-vaccination, antirHER2/neu specific antibodies showed even improved effector functions (maturation of affinity for the receptor and increased complement-derived cytotoxicity functions). HA vaccination turned out effective in both the prophylactic $(100 \%$ mice survived) and therapeutic (tumor regression in $2 / 12$ mice) settings, and broke tolerance against rHER2/neu, delaying spontaneous tumor growth in BALB-neuT mice. Both humoral and cellular responses contributed to the success of HA-based vaccination, but $\mathrm{CD}^{+} \mathrm{T}$ cells played only a marginal role.

Conclusions Cancer vaccines have not yet achieved significant clinical efficacy due to their poor immunogenicity, and the validation of more effective adjuvants occurred sometimes at the expense of safety. HA combines the unique immunomodulatory features of a TLR agonist with the tolerability of a fully natural polymer, proving to be a promising adjuvant for the creation of effective and safe cancer vaccines with the potential for rapid clinical translation.

\section{REFERENCES}

1. Dalla Pietà A, Carpanese $D$, et al. Hyaluronan is a natural and effective immunological adjuvant for protein-based vaccines. Cell Mol Immunol 2021;18(5):11971210.

2. Rosato A, Montagner IM, Carpanese D, Dalla Pietà A. Hyaluronic acid as a natural adjuvant for protein and peptide-based vaccines. 30.04.2020. WO/2020/ 084558, PCT/IB2019/059122.

Disclosure Information D. Carpanese: None. I. Montagner: None. A. Dalla Pietà: None. V. Rossi: None. A. Penna: None. G. Zuccolotto: None. G. Pasut: None. A. Grigoletto: None. A. Rosato: None.

\section{P09.04 IMPACT OF MAJOR ONCOLOGIC SURGERY ON IMMUNE RESPONSES IN THE IMMEDIATE POST-OPERATIVE SETTING IN OESOPHAGEAL ADENOCARCINOMA PATIENTS; A GUIDE TO HARNESSING THE DOUBLE- EDGED SWORD OF CANCER SURGERY}

NE Donlon*, M Davern, A Sheppard, F O'Connell, S Ramjit, C Hayes, M Mc Clean, H Temperley, C Butler, N Ravi, C Donohoe, J O' Sullivan, MR Dunne, JV Reynolds, J Lysaght. St James's Hospital, Dublin, Ireland
Background Immune checkpoint inhibitors (ICIs) are being investigated for their role as an adjunct in the multimodal treatment of oesophageal adenocarcinoma (OAC). The most appropriate time to incorporate ICIs remains unknown. Our study profiles systemic anti-tumour immunity perioperatively to help inform the optimal timing of ICIs into current standards of care for OAC patients.

Methods Systemic immunity in 11 OAC patients was phenotyped prior to oesophagectomy and on post-operative days (POD) 0, 1, 3, 7 and week 6 using flow cytometry. Longitudinal serological profiling was conducted by 54-plex-ELISA. The frequency of circulating lymphocytes, $\mathrm{T}$ cells, $\mathrm{T}$ helper cells and cytotoxic $\mathrm{T}$ lymphocytes was profiled longitudinally. The activation status of $\mathrm{T}$ cells was also assessed using CD69, CD27, CD62L and CD45RA as well as the proportion of $\mathrm{T}$ cell subsets in circulation, which included: naïve, central memory, effector memory and terminally differentiated effector memory $\mathrm{T}$ cells. This study also profiled the longitudinal alteration of immune checkpoint expression on circulating $\mathrm{T}$ cells, which included: PD-1, CTLA-4, TIGIT, TIM-3, LAG-3, PD-L1 and PD-L2. Damage-associated molecular patterns (calreticulin, HMGB1 and MIC-A/B) were also assessed.

Results The frequency of naïve $\mathrm{T}$ cells increased in circulation post-oesophagectomy from POD-0 to POD-7 $(p<0.01)$ but returned to baseline at week 6. Effector memory $\mathrm{T}$ cells had decreased by POD7 but increased substantially by week 6 $(\mathrm{p}<0.05)$. A steady increase in activated circulating CD27+ T cells was observed from POD-0 to POD-7 $(p<0.05)$. The percentage of PD-1+ and CTLA-4+ T cells peaked on POD-1 and was substantially decreased by week $6(\mathrm{p}<0.01)$. Th1 cytokines were decreased in the immediate post-operative setting with a reduction in IFN-Y, IL-12p40, CD28, CD40L and TNF Alpha. In addition to this IP-10 aka cxcl-10 which is an important chemokine ligand in recruiting anti-tumour TH1 cells and polarising the immune response to a Th1 phenotype is significantly reduced perioperatively. There is a simultaneous increase in Th2 cytokines in the immediate post-operative setting with a significant increase in IL4, IL10, IL16, IL1RA and MCP1 before returning to preoperative levels at week 6 .

Conclusion Our study highlights the prevailing immunophenotype and responses to surgery with a switch in balance towards a Th2 and potentially M2 phenotype and consequently, an immunosuppressive milieu. Therefore, orchestrating M2 reprogramming toward an M1 phenotype and similarly shifting the balance in favour of a Th1 phenotype would offer a potent therapeutic approach for augmenting tumourigenesis and promoting cancer regression. Consequently, this study paves the way for further studies and appropriate trial design are needed to interrogate the use of ICB as a trimodal approach with chemoradiotherapy and chemotherapy alone for locally advanced disease in the neoadjuvant and adjuvant setting to determine the optimal timing and subset of patients for their use in the era of precision targeted therapies.

Disclosure Information N.E. Donlon: None. M. Davern: None. A. Sheppard: None. F. O'Connell: None. S. Ramjit: None. C. Hayes: None. M. Mc Clean: None. H. Temperley: None. C. Butler: None. N. Ravi: None. C. Donohoe: None. J. O' Sullivan: None. M.R. Dunne: None. J.V. Reynolds: None. J. Lysaght: None. 